\title{
BMJ Open Melatonin, temazepam and placebo in hospitalised older patients with sleeping problems (MATCH): a study protocol of randomised controlled trial
}

\author{
Fiona Stenveld, ${ }^{\odot 1}$ Sjanne Bosman, ${ }^{\oplus 1,2}$ Barbara C van Munster, ${ }^{1,3}$ \\ Sara J Beishuizen, ${ }^{4}$ Liesbeth Hempenius, ${ }^{2}$ Nathalie van der Velde, ${ }^{4}$ \\ Nynke Smidt, ${ }^{1,5}$ Sophia E de Rooij ${ }^{1,4}$
}

To cite: Stenveld F,

Bosman S, van Munster BC, et al. Melatonin, temazepam and placebo in hospitalised older patients with sleeping problems (MATCH): a study protocol of randomised controlled trial. BMJ Open 2019;9:e025514. doi:10.1136/ bmjopen-2018-025514

- Prepublication history for this paper is available online. To view these files, please visit the journal online (http://dx.doi org/10.1136/bmjopen-2018025514).

FS and SB contributed equally.

Received 18 July 2018

Revised 14 March 2019

Accepted 19 March 2019
Check for updates

(C) Author(s) (or their employer(s)) 2019. Re-use permitted under CC BY. Published by BMJ.

For numbered affiliations see end of article.

Correspondence to

Ms. Sjanne Bosman;

s.bosman@umcg.nl

\section{ABSTRACT}

Introduction Hospitalised older patients frequently suffer from inadequate sleep, which can lead to patient distress and delayed recovery from acute illness or surgical procedure. Currently, no evidence-based treatments exist for sleeping problems in hospitalised older patients. Benzodiazepines, such as temazepam, are regularly prescribed by physicians, although they have serious side effects; for older patients in particular. Melatonin is proposed as a safe alternative for sleeping problems in hospitalised older patients, but the efficacy of melatonin is unclear in this population. Therefore, the aim of this study is to investigate the effects of melatonin and temazepam compared with placebo on sleep quality among hospitalised older patients with sleeping problems.

Methods and analysis This study is a multicentre, randomised, placebo-controlled trial. A total of 663 patients will be randomised in a 1:1:1 fashion to receive either melatonin $(n=221)$, temazepam $(n=221)$ or placebo $(n=221)$. The study population consists of hospitalised patients aged 60 years and older, with new or aggravated sleeping problems for which an intervention is needed. The primary outcome is sleep quality measured with the Leeds Sleep Evaluation Questionnaire (LSEQ). Secondary outcomes include sleep parameters measured with actigraphy and medication-related adverse effects. Ethics and dissemination This study was approved by the Medical Ethics Committee of the Academic Medical Centre Amsterdam, (No 2015_302). Study findings will be disseminated through presentations at professional and scientific conferences and publications in peer-reviewed journals.

Trial registration number NTR6908; Pre-results.

\section{INTRODUCTION}

\section{Background}

Hospitalised older patients frequently experience sleeping problems due to medical, psychological or environmental disturbances. The prevalence of sleep problems among older patients in medical and surgical wards varies between $30 \%$ and $65 \% .^{1-3}$ Apart from the feeling of tiredness, insufficient sleep
Strengths and limitations of this study

- The results of this study will provide knowledge relevant for clinical practice and fill gaps in current guidelines concerning sleep problems in hospitalised older patients.

- To optimise the generalisability of the findings, this is a multicentre study with participants from different wards and minimal exclusions criteria.

- This study investigates, next to the effects, also the adverse events of melatonin and temazepam compared with placebo.

- Patients with cognitive impairment are excluded due to ethical considerations, even though these vulnerable patients may benefit the most from the study outcomes.

has substantial consequences for hospitalised patients. This includes delayed recovery from acute illness or surgical procedures because of its immunosuppressive effect, ${ }^{45}$ risk of delirium and falls ${ }^{6-9}$ and disturbances in metabolic and endocrine functioning. ${ }^{10} 11$

Thus, adequate treatment of sleeping problems is necessary. Today, it is still unclear how sleeping problems should be treated in the hospital. Non-pharmacological interventions are advised as first choice therapy in hospitalised patients, ${ }^{12-14}$ but there is limited evidence to support the effectiveness of non-pharmacological interventions. ${ }^{15}$ With regard to pharmacological interventions, there is insufficient evidence to support its effectiveness for improving quality or quantity of sleep in hospitalised patients suffering from poor sleep. ${ }^{16}$

Currently, up to $29 \%$ of older hospitalised patients with sleeping problems receive benzodiazepines, ${ }^{1718}$ despite a recent meta-analysis of risks and benefits for short-term treatment of insomnia, which stated that benzodiazepines have little effect and may not outweigh 
the risks of adverse events, especially in a high-risk older population. ${ }^{19}$ Older patients have a higher risk of adverse drug events from benzodiazepines due to altered pharmacokinetics and pharmacodynamics. Common adverse drug events include in-hospital falls, delirium, aspiration and respiratory depression. ${ }^{20}{ }^{21}$ Furthermore, use of benzodiazepine during hospitalisation can lead to prolonged use at home, which might promote chronic use and substance-dependency. ${ }^{22}$

The neuroendocrine hormone melatonin has been proposed as a safer alternative to improve sleep quality because of its limited side effects. ${ }^{82-25}$ Melatonin, produced by the pineal gland at night, is well known to influence circadian rhythm and sleep. ${ }^{26}$ Two randomised control trials (RCTs) investigated the effectiveness of melatonin compared with placebo in older community dwelling persons with chronic insomnia and found that melatonin improved sleep quality significantly. ${ }^{27} 28$ However, studies on the effectiveness of melatonin for sleeping problems in acute hospitalised older patients are scarce. Three RCTs have been performed in adult patients on the intensive care unit, ${ }^{29-31}$ showing inconsistent results; one out of three reported a positive effect of melatonin..$^{30}$ All three studies were underpowered and none specifically assessed older patients with predefined sleep related problems.

Only one small RCT investigated the effectiveness of melatonin on a general medical ward in adults, which resulted in beneficial effects for melatonin compared with placebo on the sleep onset latency and patient satisfaction. $^{32}$

The overall aim of this study is to establish an evidencebased pharmacological treatment for hospitalised older patients with sleeping problems. In this study, we will first hypothesise that melatonin and temazepam are more effective than placebo on sleep quality among hospitalised older patients with sleeping problems and second melatonin is more effective than temazepam.

\section{Objectives}

The primary objective is to investigate first the effects of melatonin and temazepam compared with placebo among hospitalised older patients on self-reported sleep quality. Second, to investigate if melatonin is more effective than temazepam.

The secondary objectives are to investigate the effects of melatonin, temazepam and placebo on:

1. Other self-reported sleep parameters: getting to sleep, awakening from sleep, behaviour following wakefulness.

2. Objective sleep parameters: reduction in sleep onset latency, sleep efficiency, number and duration of wake bouts, time awake after sleep onset.

3. Adverse events related to study medication: incidence of delirium, cognition, number of falls, complications.

4. Length of hospital stay in days.

5. Time needed to reach improvement in self-reported sleep quality.

6. Proportion of good nights of sleep during study period.

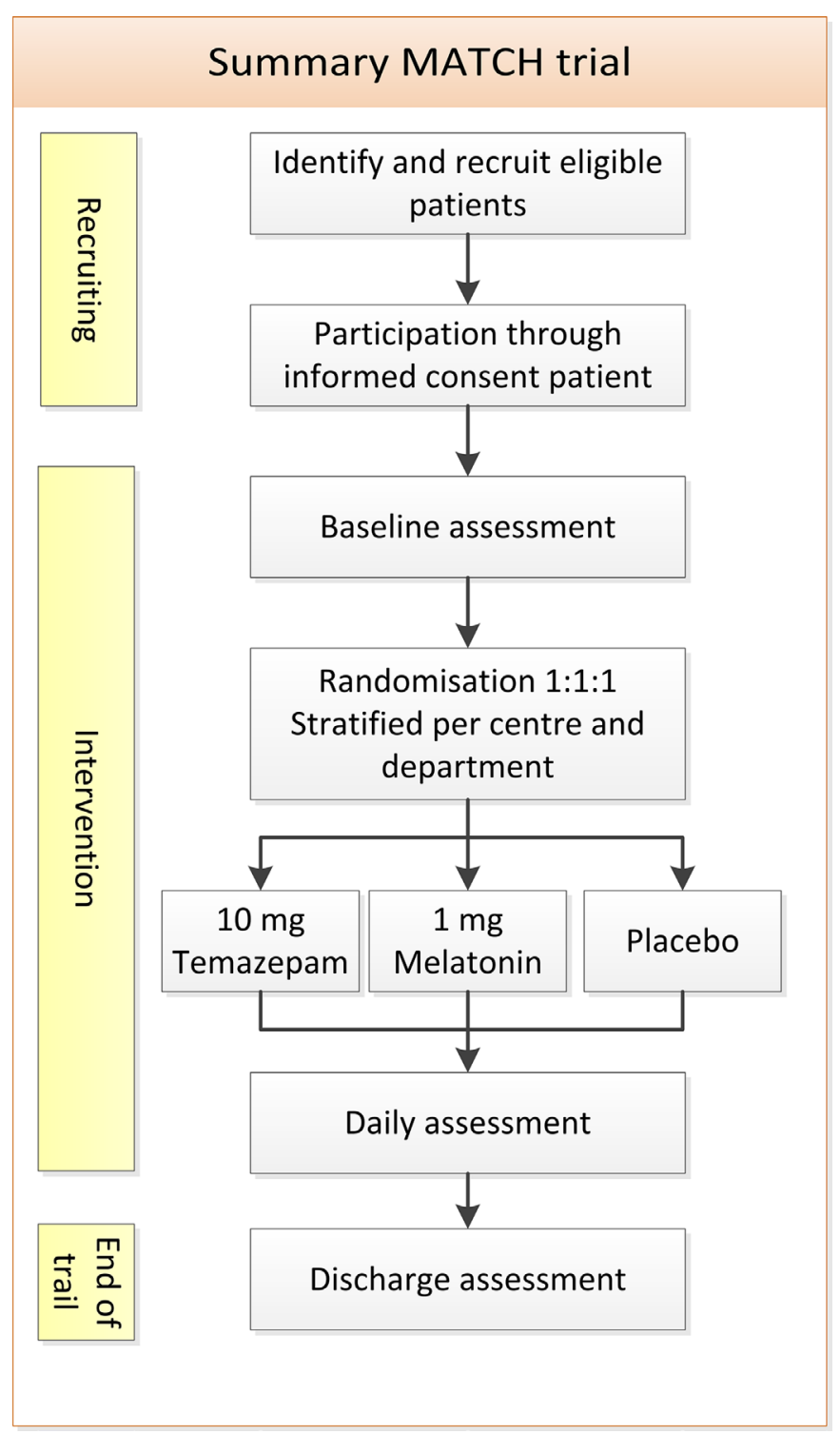

Figure 1 Flowchart of trial design summary.

\section{METHODS}

\section{Trial design}

This study is a multicentre, randomised, placebo-controlled trial. Figure 1 shows the trial design summary. This study will be conducted in two university teaching hospitals and in two teaching hospitals in the Netherlands: the Academic Medical Centre (AMC) Amsterdam, the University Medical Centre Groningen (UMCG), Gelre Hospitals Apeldoorn and the Medical Centre Leeuwarden (MCL). The design of this trial protocol has followed the recommendations of the SPIRIT 2013 statement. ${ }^{33}$

\section{Patient and public involvement}

Senior citizens were involved in several stages of this project, including the relevance of the study, developing the research question, the study design and conduct of the trial. The older representatives (Denktank 60+) partnered with us on the information material, the questionnaires and the burden of the questionnaires from a patient perspective. Once the trial has been published, 
participants will be informed of the results in a study newsletter suitable for the public.

\section{Eligibility criteria}

Eligible participants must have the following characteristics:

1. 60 years or older.

2. Admitted to the hospital for a medical or surgical reason.

3. Experiencing or complaining about new onset or aggravated sleep problems during hospital admission, for which a pharmacological intervention is needed based on the judgement of the attending physician.

4. Ability to fill out a sleep questionnaire.

People with the following characteristics are not eligible:

1. Inability to speak, understand or write Dutch.

2. Lack of decision-making capacity.

3. Previously diagnosed dementia.

4. Transferred from another hospital to one of the study centres, with insufficient information on previous use of sleep medication.

5. Expected stay in hospital of $<48$ hours.

6 . Concurrent use of sedative for a sleeping disorder (>2 dosages in the last week), such as benzodiazepine, non-benzodiazepine (Z-drugs) or melatonin.

7. Alcohol consumption >13 units/week for women and $>20$ units/week for men.

8. Drug interactions with melatonin or contraindications for benzodiazepine use.

First selection of eligible patients will be done by the attending physician, if eligible the physician will inform the research team. Final selection will be done by a member of the research team, who also will recruit eligible participants and include them after written informed consent for participation has been obtained.

\section{Randomisation and blinding}

After patients have given informed consent, participants will be allocated randomly to either temazepam, melatonin or placebo capsules (ratio is 1:1:1). Randomisation is stratified per centre and department, the strata include the four centres (AMC, Gelre hospitals Apeldoorn, MCL and UMCG) and departments (surgical vs medical). A stratified block randomisation made by an independent statistician is used and generated by the programme CASTOR Electronic Data Capture (Castor EDC, Ciwit BV, Amsterdam, the Netherlands, 2017). The researchers are not informed about the block sizes.

All medication used in the study will appear identical to ensure true blinding of the patient, attending physician, nurse and investigators. The study medication is manufactured by Tiofarma, Oud-Beijerland, the Netherlands and will be distributed to the study sites' pharmacies. Study medication must be kept intact at all times; the capsules cannot be broken. All study medication is packed in Single Unit Supply packages, blinded and dispensed by site trial pharmacists. The dispensing pharmacist will record the patient, patient ID and assigned medication by the randomisation process. Storage, delivery, dispensing and destruction of medication will adhere to Good Clinical Practice regulations (ICH-GCP). ${ }^{34}$

At the end of the study, when all data are collected, the data manager will lock the database. The information of the study arm (melatonin, temazepam and placebo) will be masked by the data manager, after which analysis can be performed in a blinded way by the researchers. Randomisation method will be broken when the study team has declared the data analysis finished.

In case of emergencies or urgent situations, such as anaphylactic reactions or loss of consciousness, and knowledge of the actual treatment is essential for further patient management, the blinding of the treatment of an individual participant can be broken (unblinded). In this case, the attending physician will contact the trial pharmacist to disclose the blinding of the study treatment for this individual patient.

\section{Intervention}

After randomisation, all participants receive the allocated medication once daily which is in the evening before sleep (ante noctem). Study medication will be administered orally starting on the day of study enrolment.

Melatonin $1 \mathrm{mg}$ and temazepam $10 \mathrm{mg}$ will be used as study medication. The dosage of $1 \mathrm{mg}$ melatonin is chosen based on a recent systematic review, to best mimic the normal physiological circadian rhythm of melatonin by avoiding prolonged supra-physiological blood levels in case of higher dosages. ${ }^{35}$ The dosage of $10 \mathrm{mg}$ temazepam is the recommend dose for older patients. ${ }^{36}$

Study medication will be continued to either a maximum of 10 days, or 12 hours before hospital discharge, or after three consecutive nights of good sleep, or until participant decides to withdraw from the study. In case sleep problems do not resolve with study medication, the dosage can be doubled. This decision will be left to the patient's attending physician. Attending physicians are instructed only to consider a double dose when the patient has slept badly for two nights in a row, in case there are no environmental or treatable physical factors that could cause the poor quality of sleep (QOS) and they should take possible adverse effects of study medication into account. Double doses of study medication will be registered in the case report form and in the medical record.

Study medication will be discontinued if a condition occurs that needs treatment with benzodiazepines (eg, anxiety), if events occur that imply a higher risk of adverse events (eg, respiratory insufficiency or starting chinolones) and in case of persisting sleeping problems. Persisting sleep problems is defined as self-reported poor QOS for three consecutive nights after study medication has been doubled.

After study medication is discontinued, either due to a reason listed above or due to end of study period, treatment will be provided according to the hospital's protocol and under the responsibility of the attending 
physician. Patients remain in the study and follow the study protocol, except for using study medication.

\section{Timing of measurement and outcome measures}

Demographic data, such as age, sex, nationality, highest educational status and living situation will be collected at baseline. Medication use, blood tests 24 hours prior to study inclusion or 24 hours after study inclusion, severity of foregoing sleep problems, depression and anxiety, level of independence, health status and vulnerability (eg, fall risk) are measured at baseline. Primary and secondary outcome measures will be recorded at baseline (T0) and every day until the 10th day (T1-T10) or until discharge. Table 1 provides an overview of the timing of measurements and the outcome measures.

\section{Primary outcome}

The primary outcome of the study is change in self-reported sleep quality during the treatment period ( $\mathrm{T} 0$ T10). Sleep quality will be assessed with the Leeds Sleep Evaluation Questionnaire (LSEQ) ${ }^{3738}$

\section{Secondary outcomes}

1. Change in self-reported sleep measures: getting to sleep, awakening from sleep and behaviour following wakefulness during treatment period will be assessed with the other subscales of the LSEQ. These measures will be assessed on T0 until T10.

2. Objective sleep parameters. To assess objective sleep parameters participants will wear a MotionWatch eight during the study period (T0-T10). This wristwatch-like device will calculate sleep latency, sleep efficiency, number and duration of wake bouts, time awake after sleep onset in minutes based on motion of the patient.

3. Number of good night sleep. Good nights of sleep will be assessed with a numeric rating scale for QOS. On the numeric rating scale $\leq 4$ is considered a bad night's sleep, 5-6 is neither a good or bad night's sleep and $\geq 7$ is a good night's sleep. ${ }^{39}$

4. Time needed to reach improvement in self-reported sleep quality will be based on the LSEQ as well. Improvement of sleep quality is defined as the first day that an improvement of $10 \mathrm{~mm}$ is reached compared with baseline.

5. Adverse events defined as confusion, delirium, reduced consciousness, falls, aspiration and other additional adverse events will be monitored daily on T0-T10.

6. Length of hospital stay in days will be recorded at discharge.

To ensure consistency and optimisation of the data collection process, all members of the research team who will include patients in the study will follow a training how to conduct measurements according to Standard Operating Procedures and how to fill out the Case Report Forms. Non-adherence to the study protocol will be obviated as much as possible. However, when it occurs this will be registered by the research team.

\section{Sample size calculation}

Sample size calculation is based on the primary outcome measure QOS, assessed with the LSEQ. To establish a clinically relevant observed effect of melatonin, temazepam or placebo a difference of $10 \mathrm{~mm}$ on the QOS was chosen. ${ }^{40}$ The SD was calculated based on the SE presented in this study. To detect a mean difference of $10 \mathrm{~mm}$, two-sided, with a power of $80 \%$ and significance level of $\alpha<0,05$, we need at least 210 patients per group (total of 630 patients).

The expected dropout rate during study period is $5 \% .^{41}$ Therefore, we should recruit at least 663 patients, with 221 patients in each study arm.

\section{Data analysis}

Data will be analysed according to an intention-to-treat principle. Researchers are not aware of the allocation of the intervention and therefore analyses will be conducted in a blinded way. A per-protocol analysis will be conducted excluding patients that did not receive the intervention according to protocol and were incorrectly included in the study. To determine the effects of temazepam, melatonin or placebo on sleep quality, linear mixed models will be used. Analysis will be performed on change of sleep quality over time taking baseline value into account (ie, night before start of study medication). The following covariates will be added to the model: age, gender, different concurrent medication (eg, morphine), severity of disease diagnosis (Rotterdam Symptom Checklist (RSCL), pain severity (visual analogue scale, RSCL), acute or elective admission to the hospital, foregoing sleep problems (insomnia severity index), number of patients in the room, level of independency (Katz Index of Independence in Activities of Daily Living), level of anxiety (Hospital Anxiety Depression scale) and days in the hospital before inclusion. Linear mixed models will also be used to evaluate getting to sleep, awakening from sleep, behaviour following wakefulness and the objective sleep parameters measured with actigraphy. Analyses of the time needed to reach improvement of sleep quality will be done with an interval-censored survival analysis. Proportion of good nights of sleep measured with the numeric ratings scale will be analysed, when normally distributed, with multiple linear regression analysis. For the analysis of adverse events related to study medication, descriptive statistics will be used to show which adverse events occurred. Depending on the occurrence of these adverse-events and distribution, log-linear analysis, Poisson or multiple regression analysis will be used. Time to occurrence of an adverse event will be analysed with Cox regression.

\section{Data management}

All participants are assigned a specific trial number, and all data will be entered in an electronic trial-specific database. The data entry process will be documented and produce an audit trail. The database will be stored and maintained by Castor EDC compliant with ICH-GCP 
Table 1 Overview of the timing of measurements and outcome measures

\begin{tabular}{|c|c|c|c|c|}
\hline \multirow[b]{2}{*}{ Measurement } & \multirow[b]{2}{*}{ Description and instrument } & \multicolumn{3}{|c|}{ Timepoints } \\
\hline & & Baseline & Daily & Discharge \\
\hline \multirow[t]{2}{*}{ Sleep quality } & $\begin{array}{l}\text { Leeds Sleep Evaluation Questionnaire (LSEQ), }{ }^{384} \text { 10-item } \\
\text { questionnaire, self-rated, on 0-100 mm line. Validated tool for } \\
\text { measuring changes in sleep on four domains; getting to sleep, quality } \\
\text { of sleep, ease and awakening from sleep and alertness and behaviour } \\
\text { following wakefulness } \\
\text { The Dutch version of the LSEQ is forwards and backwards translated } \\
\text { according to the translation guidelines }\end{array}$ & $x$ & $x$ & \\
\hline & $\begin{array}{l}\text { Numeric rating scale for overall sleep, an overall numeric rating of } \\
\text { quality of sleep. With '0' representing a very bad night's sleep and '10' } \\
\text { representing a very good night's sleep }\end{array}$ & $x$ & $x$ & \\
\hline Sleep pattern & $\begin{array}{l}\text { The core consensus sleep diary }{ }^{46} \text {, a } 10 \text {-item sleep log to gain insight in } \\
\text { the sleep pattern of a participant }\end{array}$ & $\mathrm{x}$ & $x$ & \\
\hline $\begin{array}{l}\text { Factor } \\
\text { influencing sleep }\end{array}$ & $\begin{array}{l}\text { Based on literature, }{ }^{15} 47-50 \text { an overview is made of } 10 \text { influencing factors } \\
\text { on sleep in hospitals. Participants can indicate whether these factors } \\
\text { influenced their sleep and if so, to what extent }\end{array}$ & $x$ & $x$ & \\
\hline $\begin{array}{l}\text { Objective sleep } \\
\text { parameters }\end{array}$ & $\begin{array}{l}\text { Actigraphy, MotionWatch } 8 \text {, a wristwatch-like device, worn on the non- } \\
\text { dominant hand. The actiwatch registers motion in epochs of } 30 \text { s. Based } \\
\text { on motion sleep latency, sleep efficiency, number and duration of wake } \\
\text { bouts, time awake after sleep onset in minutes can be calculated }\end{array}$ & $x$ & $x$ & \\
\hline \multirow[t]{3}{*}{ Cognition } & $\begin{array}{l}\text { Mini-Mental State Examination }{ }^{51} \text {, a } 10 \text {-item screening tool to evaluate } \\
\text { global cognitive function }\end{array}$ & $x$ & $x^{*}$ & \\
\hline & $\begin{array}{l}\text { Stroop Colour-Word test }{ }^{52}{ }^{53} \text {, consists of three subtasks. Used to } \\
\text { measure attention, processing speed, cognitive flexibility and working } \\
\text { memory }\end{array}$ & $\mathrm{x}$ & $x^{*}$ & \\
\hline & $\begin{array}{l}\text { The Trail Making Test (TMT) A\&B }{ }^{54}{ }^{55} \text {, consists of two subtasks, is used } \\
\text { to measure visual search, attention, divided attention, processing speed } \\
\text { and cognitive flexibility }\end{array}$ & $x$ & $x$ & \\
\hline \multirow[t]{2}{*}{$\begin{array}{l}\text { Assessment of } \\
\text { delirium }\end{array}$} & $\begin{array}{l}4 A T^{56} \text { a four-item screening tool to detect attention deficits due to } \\
\text { delirium }\end{array}$ & $x$ & $x \dagger$ & \\
\hline & $\begin{array}{l}D S M-V V^{57} \text { the Diagnostic and Statistical Manual of the American } \\
P s y c h i a t r i c ~ A s s o c i a t i o n(D S M-5) \text { the most widely used nomenclature by } \\
\text { clinicians and researchers for the classification of delirium }\end{array}$ & $x \neq$ & $x \neq$ & \\
\hline $\begin{array}{l}\text { Severity of sleep } \\
\text { problems }\end{array}$ & $\begin{array}{l}\text { Insomnia severity index, }{ }^{58} \text { a seven-item questionnaire, self-rated, to } \\
\text { quantify perceived insomnia severity }\end{array}$ & $x$ & & \\
\hline $\begin{array}{l}\text { Depression and } \\
\text { anxiety }\end{array}$ & $\begin{array}{l}\text { Hospital Anxiety Depression scale }{ }^{59} \text {, a } 14 \text {-item questionnaire, self-rated, } \\
\text { consisting of two seven-item subscales looking at depression and } \\
\text { anxiety }\end{array}$ & $x$ & & \\
\hline $\begin{array}{l}\text { Level of } \\
\text { independence }\end{array}$ & $\begin{array}{l}\text { Katz-15 Activities of Daily Living }(A D L) \text { index scale, }{ }^{6061} \text { the modified Katz } \\
\text { ADL index 15-item scale, answered with 'yes' or' no' to evaluate the } \\
\text { level of independence }\end{array}$ & $\mathrm{x}$ & & \\
\hline \multirow[t]{2}{*}{ Health status } & $\begin{array}{l}\text { Rotterdam symptom check list }(R S C L)^{62} \text {, a } 30 \text {-item questionnaire, self- } \\
\text { rated, to assess physical and psychological symptom }\end{array}$ & $x$ & $x \S$ & \\
\hline & $\begin{array}{l}\text { Charlson comorbidity index (CCl), }{ }^{63} \text { a classification method for comorbid } \\
\text { conditions. It is a valid method to estimate risk of death comorbid } \\
\text { disease in } 1 \text { year }\end{array}$ & & & $x$ \\
\hline $\begin{array}{l}\text { Vulnerability (eg, } \\
\text { fall risk) }\end{array}$ & Safety management system patient screening ${ }^{64}$ & $x$ & & \\
\hline Adverse events & $\begin{array}{l}\text { Confusion, delirium, reduced consciousness, falls, aspiration, other } \\
\text { additional adverse events (in complication register) and length of } \\
\text { hospital stay in days }\end{array}$ & & $x$ & \\
\hline \multicolumn{5}{|c|}{$\begin{array}{l}\text { *On day } 3 \text { Stroop Colour-Word test and TMT are repeated with daily assessment. } \\
\text { †Administered if a delirium is suspected. } \\
\text { †When score on } 4 \text { AT deviates, detailed assessment of mental status with DSM-V to reach diagnosis of delirium. } \\
\text { §On day } 4 \text { RSCL is repeated with daily assessment. }\end{array}$} \\
\hline
\end{tabular}


regulations $^{34}$ and the European Data Protection Directive. Confidentiality of participant information will be maintained throughout the trial. Results can only be traced to participants by the researchers involved. Data will be stored for a period of 15 years after the study has ended, according to ICH-GCP regulations. ${ }^{34}$

\section{Participant safety and monitoring}

This trial is considered a low-risk study. Melatonin in a dosage of $1 \mathrm{mg}$ is not known to show any adverse events and is considered safe for use by older patients. Temazepam has potential adverse-effects but this medication is part of usual treatment of hospitalised older patients with sleep problems, participation in the study will not expose patients to any additional risks. Close monitoring of all possible adverse events related to study medication will take place, and in case any adverse events occur, appropriate measures will be taken in consultation with the attending physician. The Clinical Research Unit (CRU) of the AMC will conduct the monitoring of this trial in compliance with the Medical Research Involving Human Subjects Act (WMO) and ICH-GCP regulations. ${ }^{34}$ Annual reports of the study progress will be sent to the Medical Research Ethics Committee of the Academic Medical Centre Amsterdam. As this trial is considered as a low risk study with a low risk on serious adverse events, no Data and Safety Monitoring Board (DSMB) will be installed or interim analysis performed.

\section{DISCUSSION}

Although sleeping problems are a common complaint in hospitalised older patients, there is still no evidence based pharmacological treatment available. Most studies on (non-) pharmacological treatment of sleeping problems in older patients were performed in community dwelling older people. However, sleep quality in hospitalised elderly is influenced by other factors than community dwelling older people and might require other therapeutic regimes. ${ }^{42}$ For example, light and noise in the room influences the QOS in hospitalised older patients and it is difficult to measure, subsequently standardise for these confounders on the ward. In order to minimise the effect of these potential confounders, we stratified randomisation, in which patients are randomised by hospitals and departments. In this way, the known and unknown confounders are randomised and equally distributed among the groups.

In this study it was not possible to measure sleep quality and quantity using polysomnography, which is the gold standard to measure sleep quality and quantity. Instead of polysomnography, we measured objective sleep parameters indirectly by using an actigraphy, in addition to the self-reported sleep outcome measures.

This study is the first large randomised controlled trial on the effects of melatonin, temazepam or placebo in hospitalised older patients, which aimed to establish an evidence based pharmacological treatment for sleeping problems in older hospitalised patients. Irrespective of the results of this trial, the outcomes can be used for developing clinical guidelines for the treatment of sleep problems among hospitalised older patients.

\section{ETHICS AND DISSEMINATION}

The trial will be conducted in accordance with the Declaration of Helsinki 1996 and principles of good clinical practice. Any substantial protocol amendments will be submitted to the ethics committee. A register of protocol amendments will be made available in the study protocol.

The grant was an unrestricted grant from the University of Amsterdam Foundation.

Trial methods and results will be reported according to the Consolidated Standards of Reporting Trials 2010 guidelines. ${ }^{43}$ The study findings will be published in relevant peer-reviewed journals.

\section{Trial status}

The trial has started on 29th of January 2018.

\section{Author affiliations}

${ }^{1}$ Department of Geriatric Medicine, University of Groningen, University Medical Centre Groningen, Groningen, The Netherlands

${ }^{2}$ Department of Geriatric Medicine, Medical Centre Leeuwarden, Leeuwarden, The Netherlands

${ }^{3}$ Department of Geriatrics, Gelre Hospitals, Apeldoorn, The Netherlands ${ }^{4}$ Department of Internal Medicine, Geriatrics Section, Amsterdam Public Health, Academic Medical Centre, Amsterdam, The Netherlands

${ }^{5}$ Department of Epidemiology, University of Groningen, University Medical Centre, Groningen, The Netherlands

Correction notice This article has been corrected since it first published online. The open access licence type has been amended.

Acknowledgements The authors thank Carolien Jansen (data manager) and the senior citizens of Denktank 60+ Noord for their time, critical appraisal and advise.

Contributors FS and SB contributed equally to this paper. SDR is responsible for the original conception of the study and obtaining the grant. FS, SB, SJB, NS, BCvM, NvdV, LH and SDR attributed to the design of the study. FS and SB wrote the first draft of the manuscript, all authors contributed to further drafts. All authors read and approved the final draft.

Funding The MATCH-trial is unrestrictedly financed by the Dalderup foundation. Competing interests None declared.

Patient consent for publication Not required.

Ethics approval This study was approved by the Medical Research Ethics Committee of the Academic Medical Centre Amsterdam in June 2017. Reference number: 2015_302. The executive boards of the other participating centres have provided local feasibility approval.

Provenance and peer review Not commissioned; externally peer reviewed.

Open access This is an open access article distributed in accordance with the Creative Commons Attribution 4.0 Unported (CC BY 4.0) license, which permits others to copy, redistribute, remix, transform and build upon this work for any purpose, provided the original work is properly cited, a link to the licence is given, and indication of whether changes were made. See: https://creativecommons.org/ licenses/by/4.0/.

\section{REFERENCES}

1. Enomoto M, Tsutsui T, Higashino S, et al. Sleep-related problems and use of hypnotics in inpatients of acute hospital wards. Gen Hosp Psychiatry 2010;32:276-83.

2. Isaia G, Corsinovi L, Bo M, et al. Insomnia among hospitalized elderly patients: prevalence, clinical characteristics and risk factors. Arch Gerontol Geriatr 2011;52:133-7. 
3. Meissner HH, Riemer A, Santiago SM, et al. Failure of physician documentation of sleep complaints in hospitalized patients. West $J$ Med 1998;169:146-9.

4. Irwin M, McClintick J, Costlow C, et al. Partial night sleep deprivation reduces natural killer and cellular immune responses in humans. Faseb J 1996;10:643-53.

5. Weinhouse GL. When perception may not meet reality. Crit Care Med 2015;43:248-9

6. Inouye SK, Westendorp RG, Saczynski JS. Delirium in elderly people. Lancet 2014;383:911-22.

7. Watson PL, Ceriana P, Fanfulla F. Delirium: is sleep important? Best Pract Res Clin Anaesthesiol 2012;26:355-66.

8. Chen S, Shi L, Liang F, et al. Exogenous Melatonin for Delirium Prevention: a Meta-analysis of Randomized Controlled Trials. Mol Neurobiol 2016;53:4046-53.

9. Wu L, Sun D. Sleep duration and falls: a systemic review and metaanalysis of observational studies. J Sleep Res 2017;26:293-301.

10. Leproult R, Copinschi G, Buxton O, et al. Sleep loss results in an elevation of cortisol levels the next evening. Sleep 1997:20:865-70.

11. Kamdar BB, Needham DM, Collop NA. Sleep deprivation in critical illness: its role in physical and psychological recovery. $J$ Intensive Care Med 2012;27:97-111.

12. Nederlande Internisten vereniging. Evidence-based richtlijn nieuwe of verergerende slaapproblemen bij acuut opgenomen ouderen in het ziekenhuis of verpleeghuis. Richtlijn 2015:1-89.

13. Young JS, Bourgeois JA, Hilty DM, et al. Sleep in hospitalized medical patients, part 2: behavioral and pharmacological management of sleep disturbances. J Hosp Med 2009;4:50-9.

14. Lenhart SE, Buysse DJ. Treatment of insomnia in hospitalized patients. Ann Pharmacother 2001;35:1449-57.

15. Tamrat R, Huynh-Le MP, Goyal M. Non-pharmacologic interventions to improve the sleep of hospitalized patients: a systematic review. $J$ Gen Intern Med 2014;29:788-95.

16. Kanji S, Mera A, Hutton B, et al. Pharmacological interventions to improve sleep in hospitalised adults: a systematic review. BMJ Open 2016;6:e012108.

17. Frighetto L, Marra C, Bandali S, et al. An assessment of quality of sleep and the use of drugs with sedating properties in hospitalized adult patients. Health Qual Life Outcomes 2004;2:17.

18. Gillis CM, Poyant JO, Degrado JR, et al. Inpatient pharmacological sleep aid utilization is common at a tertiary medical center. $J$ Hosp Med 2014;9:652-7.

19. Glass J, Lanctôt KL, Herrmann N, et al. Sedative hypnotics in older people with insomnia: meta-analysis of risks and benefits. BMJ 2005;331:1169.

20. Cumming RG, Le Couteur DG. Benzodiazepines and risk of hip fractures in older people: a review of the evidence. CNS Drugs 2003;17:825-37.

21. Brandt J, Leong C. Benzodiazepines and Z-Drugs: An Updated Review of Major Adverse Outcomes Reported on in Epidemiologic Research. Drugs R D 2017;17:493-507.

22. Halfens R, Cox K, Kuppen-Van Merwijk A. Effect of the use of sleep medication in Dutch hospitals on the use of sleep medication at home. J Adv Nurs 1994;19:66-70.

23. Buscemi N, Vandermeer B, Hooton N, et al. The efficacy and safety of exogenous melatonin for primary sleep disorders. A meta-analysis. $J$ Gen Intern Med 2005;20:1151-8.

24. de Jonghe A, Korevaar JC, van Munster BC, et al. Effectiveness of melatonin treatment on circadian rhythm disturbances in dementia. Are there implications for delirium? A systematic review. Int $J$ Geriatr Psychiatry 2010;25:1201-8.

25. Chakraborti D, Tampi DJ, Tampi RR. Melatonin and melatonin agonist for delirium in the elderly patients. Am J Alzheimers Dis Other Demen 2015;30:119-29.

26. Brzezinski A. Melatonin in humans. N Engl J Med 1997;336:186-95.

27. Wade AG, Ford I, Crawford G, et al. Efficacy of prolonged release melatonin in insomnia patients aged 55-80 years: quality of sleep and next-day alertness outcomes. Curr Med Res Opin 2007;23:2597-605.

28. Lemoine $\mathrm{P}, \mathrm{Nir} \mathrm{T}$, Laudon $\mathrm{M}$, et al. Prolonged-release melatonin improves sleep quality and morning alertness in insomnia patients aged 55 years and older and has no withdrawal effects. J Sleep Res 2007:16:372-80

29. Bourne RS, Mills GH, Minelli C. Melatonin therapy to improve nocturnal sleep in critically ill patients: encouraging results from a small randomised controlled trial. Crit Care 2008;12:R52.

30. Shilo L, Dagan Y, Smorjik Y, et al. Effect of melatonin on sleep quality of COPD intensive care patients: a pilot study. Chronobiol Int 2000;17:71-6.
31. Ibrahim MG, Bellomo R, Hart GK, et al. A double-blind placebocontrolled randomised pilot study of nocturnal melatonin in tracheostomised patients. Crit Care Resusc 2006;8:187-91.

32. Andrade C, Srihari BS, Reddy KP, et al. Melatonin in medically ill patients with insomnia: a double-blind, placebo-controlled study. $J$ Clin Psychiatry 2001;62:41-5.

33. Agha RA, Altman DG, Rosin D. The SPIRIT 2013 statement--defining standard protocol items for trials. Int J Surg 2015;13:288-91.

34. ICH. Integrated addendum to ICH e6(R1): Guideline for good clinical practice $\mathrm{ICH}, 2016$.

35. Vural EM, van Munster BC, de Rooij SE. Optimal dosages for melatonin supplementation therapy in older adults: a systematic review of current literature. Drugs Aging 2014;31:441-51.

36. Zorginstituut Nederland. Temazepam benzodiazepine agonisten | N05CD07. https://www.farmacotherapeutischkompas.nl/bladeren/ preparaatteksten/t/temazepam (accessed 4 Nov 2017).

37. Parrott AC, Hindmarch I. The Leeds Sleep Evaluation Questionnaire in psychopharmacological investigations - a review. Psychopharmacology 1980;71:173-9.

38. Tarrasch R, Laudon M, Zisapel N. Cross-cultural validation of the Leeds sleep evaluation questionnaire (LSEQ) in insomnia patients. Hum Psychopharmacol 2003;18:603-10.

39. Van Beuningen J, van der Houwen K, Moonen L. Measuring wellbeing An analysis of different response scales. The Hague/Heerlen 2014 www.cbs.nl

40. Zisapel N, Nir T. Determination of the minimal clinically significant difference on a patient visual analog sleep quality scale. J Sleep Res 2003:12:291-8.

41. de Jonghe A, van Munster BC, Goslings JC, et al. Effect of melatonin on incidence of delirium among patients with hip fracture: a multicentre, double-blind randomized controlled trial. CMAJ 2014;186:E547-56

42. Wesselius HM, van den Ende ES, Alsma J, et al. Quality and Quantity of Sleep and Factors Associated With Sleep Disturbance in Hospitalized Patients. JAMA Intern Med 2018;178:1201-71.

43. Moher D, Hopewell S, Schulz KF, et al. CONSORT 2010 explanation and elaboration: updated guidelines for reporting parallel group randomised trials. Int J Surg 2012;10:28-55.

44. Parrott AC, Hindmarch I. Factor analysis of a sleep evaluation questionnaire. Psychol Med 1978;8:325-9.

45. Beaton DE, Bombardier C, Guillemin F, et al. Guidelines for the process of cross-cultural adaptation of self-report measures. Spine 2000;25:3186-91.

46. Carney CE, Buysse DJ, Ancoli-Israel S, et al. The consensus sleep diary: standardizing prospective sleep self-monitoring. Sleep 2012;35:287-302.

47. Park MJ, Kim KH. What affects the subjective sleep quality of hospitalized elderly patients? Geriatr Gerontol Int 2017;17:471-9.

48. Bano M, Chiaromanni F, Corrias M, et al. The influence of environmental factors on sleep quality in hospitalized medical patients. Front Neurol 2014;5:267.

49. Ersser S, Wiles A, Taylor H, et al. The sleep of older people in hospital and nursing homes. J Clin Nurs 1999;8:360-8.

50. Dobing S, Frolova N, McAlister F, et al. Sleep Quality and Factors Influencing Self-Reported Sleep Duration and Quality in the General Internal Medicine Inpatient Population. PLoS One 2016:11:e0156735-6.

51. Folstein MF, Folstein SE, McHugh PR. "Mini-mental state". A practical method for grading the cognitive state of patients for the clinician. J Psychiatr Res 1975;12:189-98.

52. Stroop JR. Studies of interference in serial verbal reactions. J Exp Psychol 1935;18:643-62.

53. Hammes J. De stroop Kleur-woord test: handleiding [The Stroop Color-word test: manual]: Lisse, 1997.

54. Army Individual Test Battery. Manual of directions and scoring. Washington, DC: War Dep Adjut Gen Off, 1944.

55. Lezak DBH MD. Neuropsychological assessment. 4th edn. New York: Oxford University Press, 2004.

56. Bellelli G, Morandi A, Davis DH, et al. Validation of the 4AT, a new instrument for rapid delirium screening: a study in 234 hospitalised older people. Age Ageing 2014;43:496-502.

57. American Psychiatric Association. Diagnostic and statistical manual of mental disorders. 5th edn. Washington: DC, 2013.

58. Bastien $\mathrm{CH}$, Vallières $\mathrm{A}$, Morin $\mathrm{CM}$. Validation of the Insomnia Severity Index as an outcome measure for insomnia research. Sleep Med 2001;2:297-307.

59. Cosco TD, Doyle F, Ward M, et al. Latent structure of the Hospital Anxiety And Depression Scale: a 10-year systematic review. $J$ Psychosom Res 2012;72:180-4.

60. Laan W, Zuithoff NP, Drubbel I, et al. Validity and reliability of the Katz-15 scale to measure unfavorable health outcomes 
in community-dwelling older people. J Nutr Health Aging 2014;18:848-54.

61. Weinberger M, Samsa GP, Schmader K, et al. Comparing proxy and patients' perceptions of patients' functional status: results from an outpatient geriatric clinic. J Am Geriatr Soc 1992;40:585-8.

62. de Haes JC, van Knippenberg FC, Neijt JP. Measuring psychological and physical distress in cancer patients: structure and application of the Rotterdam Symptom Checklist. Br J Cancer 1990;62:1034-8.
63. Charlson ME, Pompei P, Ales KL, et al. A new method of classifying prognostic comorbidity in longitudinal studies: development and validation. J Chronic Dis 1987;40:373-83.

64. Oud FM, de Rooij SE, Schuurman T, et al. [Predictive value of the VMS theme 'Frail elderly': delirium, falling and mortality in elderly hospital patients]. Ned Tijdschr Geneeskd 2015;159:A8491. 